\title{
Predictors and consequences of anaemia among antiretroviral- naïve HIV-infected and HIV-uninfected children in Tanzania
}

\author{
Anirban Chatteriee ${ }^{1, *}+$, Ronald J Bosch ${ }^{2}$, Roland Kupka ${ }^{3}$, David J Hunter ${ }^{1,3}$, Gernard I \\ Msamanga ${ }^{4}$ and Wafaie W Fawzi ${ }^{1,3}$ \\ 'Department of Epidemiology, Harvard School of Public Health, Boston, MA, USA: ${ }^{2}$ Department of Biostatistics, \\ Harvard School of Public Health, Boston, MA, USA: ${ }^{3}$ Department of Nutrition, Harvard School of Public Health, \\ Boston, MA, USA: ${ }^{4}$ Department of Community Health Sciences, Muhimbili University College of Health Sciences, \\ Dar es Salaam, Tanzania
}

Submitted 22 October 2008: Accepted 1 June 2009: First published online 4 August 2009

\begin{abstract}
Objective: Predictors and consequences of childhood anaemia in settings with high HIV prevalence are not well known. The aims of the present study were to identify maternal and child predictors of anaemia among children born to HIV-infected women and to study the association between childhood anaemia and mortality.

Design: Prospective cohort study. Maternal characteristics during pregnancy and $\mathrm{Hb}$ measurements at 3-month intervals from birth were available for children. Information was also collected on malaria and HIV infection in the children, who were followed up for survival status until 24 months after birth.

Setting: Dar es Salaam, Tanzania.

Subjects: The study sample consisted of 829 children born to HIV-positive women. Results: Advanced maternal clinical HIV disease (relative risk (RR) for stage $\geq 2$ $v$. stage $1: 1 \cdot 31,95 \%$ CI $1 \cdot 14,1 \cdot 51)$ and low CD 4 cell counts during pregnancy (RR for $<350$ cells $/ \mathrm{mm}^{3} v$. $\geq 350$ cells $/ \mathrm{mm}^{3}: 1 \cdot 58,95 \%$ CI $\left.1 \cdot 05,2 \cdot 37\right)$ were associated with increased risk of anaemia among children. Birth weight $<2500 \mathrm{~g}$, preterm birth $(<34$ weeks), malaria parasitaemia and HIV infection in the children also increased the risk of anaemia. Fe-deficiency anaemia in children was an independent predictor of mortality in the first two years of life (hazard ratio $1 \cdot 99,95 \%$ CI $1 \cdot 06,3 \cdot 72$ ).

Conclusions: Comprehensive care including highly active antiretroviral therapy to eligible HIV-infected women during pregnancy could reduce the burden of anaemia in children. Programmes for the prevention of mother-to-child transmission of HIV and antimalarial treatment to children could improve child survival in settings with high HIV prevalence.
\end{abstract}

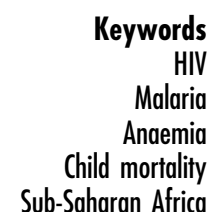

Sub-Saharan Africa
Anaemia is a major public health problem in most developing countries. Globally, anaemia is present in approximately $43 \%$ of children under the age of 4 years ${ }^{(1)}$ with close to $75 \%$ of children under 5 years of age suffering from anaemia in East Africa ${ }^{(2)}$. Studies from subSaharan Africa have reported anaemia prevalence ranging from $42 \%$ to $75 \%$ among HIV-infected children ${ }^{(3)}$. Fedeficiency anaemia is the commonest form of anaemia, and is estimated to constitute approximately $50 \%$ of the total burden of anaemia albeit with wide geographic variation $^{(4-6)}$. It is a risk factor for delayed psychomotor development and cognitive function and also impairs

$\uparrow$ Correspondence address: c/o Wafaie Fawzi, Department of Nutrition, Harvard School of Public Health, 677 Huntington Avenue, Boston, MA 02115, USA. cell-mediated immunity ${ }^{(7-10)}$. Because of the long-term effects of Fe-deficiency anaemia during infancy it is important to prevent and treat it appropriately as early in infancy as possible. Little is known about the association between maternal factors during pregnancy among HIVinfected women and the development of anaemia in their children. Maternal factors that are treatable or preventable could be potential targets for public health interventions aimed at reducing the burden of anaemia in children.

Among HIV-infected adults, anaemia is known to be a risk factor for mortality in studies from both developed and developing countries ${ }^{(11,12)}$. Little is known about the association between anaemia and mortality in HIVinfected and uninfected children born to HIV-infected women in sub-Saharan Africa. We examined the associations between maternal and child factors and development 
of anaemia in children until 24 months of age. We also studied the relationship between anaemia in HIV-infected and uninfected children born to HIV-infected women and child mortality in the first two years of life.

\section{Experimental methods}

The current prospective study was conducted in Dar es Salaam, Tanzania within the setting of a randomized trial of vitamin supplementation of HIV-infected women during pregnancy and lactation. Details of the trial have been published elsewhere ${ }^{(13)}$. HIV-infected women were between 12 and 27 weeks of gestation (mean 20 (SD 3) weeks) and, after giving informed consent, were randomized in a double-blind factorial design to one of four arms: vitamin $\mathrm{A}$, vitamin $\mathrm{A}$ and multivitamins, multivitamins excluding vitamin A or placebo. All women received standard antenatal care including daily supplementation with Fe and folic acid as well as weekly doses of chloroquine phosphate as per national guidelines in Tanzania. Women in the vitamin A arm received another high dose of vitamin A at delivery while others received a placebo. Antiretroviral therapy was not yet widely available in Tanzania during the period when the study was conducted and none of the mothers or children received antiretroviral therapy during the course of the study.

At the time of enrolment detailed background information including educational levels were collected from all women using standardized questionnaires administered by trained research nurses, who also measured the women's weight, height and left mid upper-arm circumference using calibrated instruments and standardized techniques ${ }^{(14)}$. Detailed medical information was collected and a complete physical examination including vaginal examination was conducted by trained physicians. The women provided blood samples for determination of $\mathrm{Hb}, \mathrm{CD} 4 \mathrm{~T}$-lymphocyte count and infection with Plasmodium falciparum malaria, as well stool samples for intestinal parasitic infestation. Birth weight was measured to the nearest $10 \mathrm{~g}$ using a standard beam balance (model 725; Seca, Hamburg, Germany) immediately after birth and gestational age was evaluated on the basis of maternal recall of last menstrual period. Children born to women enrolled in the trial were followed from birth through monthly visits to the study clinics; home visits were made in cases where a scheduled clinic visit was missed. The children provided blood samples at birth and every 3 months thereafter for determination of $\mathrm{Hb}$ and erythrocyte morphology as well as infection with $P$. falciparum malaria. CD4 T-lymphocyte counts were determined at birth and every 6 months thereafter, while HIV infection status was determined at birth, at 6 weeks and then at 3-month intervals.

$\mathrm{Hb}$ was measured using a CBC5 Coulter counter (Coulter Corporation, Miami, FL, USA). However, due to machine breakdown, the method was changed to the cyanmethaemoglobin method using a colorimeter (Corning, Corning, NY, USA) in the later part of the study. Erythrocyte morphology was examined in thin blood films stained with Leishman's stain. For the analysis reported in the present paper, we considered the erythrocyte morphology to be microcytic and hypochromic if $>75 \%$ of the cells examined showed microcytosis and hypochromasia. Infection with $P$. falciparum malaria was identified using Giemsa-stained thick and thin blood films. Parasite density was estimated by counting the number of parasites in 300 leucocytes and assuming a total leucocyte count of $8000 / \mathrm{mm}^{3}$ blood. Measurements of absolute counts of CD4 T cells were done using the FACScan and FACScount system (Beckton-Dickinson, San Jose, CA, USA). HIV-1 infection was diagnosed in the children on the basis of a positive peripheral mononuclear cell specimen by PCR before 18 months of age (using the Amplicor HIV-1 detection kit; Roche Diagnostics, Branchburg, NJ, USA) or a positive ELISA confirmed by a Western blot at or after 18 months of age. The time of HIV infection was designated as the midpoint between the last negative and the first positive HIV test. To the 1078 HIV-infected women enrolled in the trial, 984 live-born children were delivered. The study population consisted of 829 singleton children who had at least one $\mathrm{Hb}$ measurement available. There was no difference in the background characteristics of the 829 live-born children who were included in the analysis and those who were not included due to non-availability of at least one $\mathrm{Hb}$ measurement. For the present analysis child anaemia was defined in two different ways: anaemia $(\mathrm{Hb}<8.5 \mathrm{~g} / \mathrm{dl})$ and anaemia suggestive of Fe deficiency $(\mathrm{Hb}<8.5 \mathrm{~g} / \mathrm{dl}$ and hypochromic microcytic erythrocyte morphology with $>75 \%$ cells showing hypochromasia and microcytosis).

Generalized estimating equations (GEE; PROC GENMOD in the SAS statistical software package version 9.1 (SAS Institute, Cary, NC, USA)) were used to examine the association between maternal and infant factors and child anaemia and child anaemia suggestive of Fe deficiency separately $^{(15)}$. A GEE model with exchangeable working covariance structure was used and a binomial distribution was specified. All covariates with $P \leq 0 \cdot 20$ in the univariate models were entered into a multivariate model. Cox proportional hazards models (PROC PHREG in SAS) were fit to examine the association between child anaemia and mortality until 24 months of age. Child anaemia was defined in the same manner as for the analysis above and entered into univariate and multivariate models as a time-dependent covariate. The missing indicator method was used for covariates with missing data ${ }^{(16)}$.

The study protocol was approved by the Research and Publications Committee of Muhimbili University College of Health Sciences, the Ethical Committee of the National AIDS Control Program of the Tanzanian Ministry of Health, and the Institutional Review Board of the Harvard School of Public Health. 


\section{Results}

A total of $3351 \mathrm{Hb}$ measurements were available from 829 children over the 24 months of follow-up for an average of four measurements per child. The mean $\mathrm{Hb}$ level over all measurement occasions was $9 \cdot 99 \mathrm{~g} / \mathrm{dl}$ and the median was
$9 \cdot 70 \mathrm{~g} / \mathrm{d}(25 \mathrm{th}, 75$ th percentile: $8 \cdot 6,11 \cdot 2 \mathrm{~g} / \mathrm{dl})$. Twenty-three per cent of the $\mathrm{Hb}$ measurements were below $8 \cdot 5 \mathrm{~g} / \mathrm{dl}$.

Maternal WHO HIV clinical disease stage $\geq 2$ during pregnancy as compared with stage 1 was significantly associated with increased risk of anaemia in children up to 24 months of age (relative risk (RR) $1 \cdot 31 ; 95 \%$ CI 1.14, 1.51; Table 1 ).

Table 1 Maternal and child factors in relation to anaemia $(\mathrm{Hb}<8.5 \mathrm{~g} / \mathrm{dl})$ among children born to HIV-infected women, Dar es Salaam, Tanzania

\begin{tabular}{|c|c|c|c|c|c|c|c|c|c|}
\hline & \multicolumn{2}{|c|}{ Children at risk } & \multirow{2}{*}{$\begin{array}{c}\text { Anaemia rate } \\
\text { (/child-year) }\end{array}$} & \multirow{2}{*}{$\begin{array}{l}\text { Univariate } \\
\text { relative risk }\end{array}$} & \multirow[b]{2}{*}{$95 \% \mathrm{Cl}$} & \multirow[b]{2}{*}{$P$ value* } & \multirow{2}{*}{$\begin{array}{l}\text { Adjusted } \\
\text { relative riskt }\end{array}$} & \multirow[b]{2}{*}{$95 \% \mathrm{Cl}$} & \multirow[b]{2}{*}{$P$ valuet } \\
\hline & $n$ & $\%$ & & & & & & & \\
\hline \multicolumn{10}{|l|}{ Maternal predictors } \\
\hline \multicolumn{10}{|l|}{ HIV disease stage } \\
\hline$\geq 2$ & 158 & 19 & $1 \cdot 15$ & $1 \cdot 33$ & $1 \cdot 15,1 \cdot 54$ & 0.0001 & $1 \cdot 31$ & $1 \cdot 14,1 \cdot 51$ & 0.0001 \\
\hline 1 & 666 & 81 & $0 \cdot 87$ & $1 \cdot 00$ & & & $1 \cdot 00$ & & \\
\hline \multicolumn{10}{|c|}{ CD4 cell count $\left(/ \mathrm{mm}^{3}\right)$} \\
\hline$<350$ & 293 & 38 & 0.96 & 1.06 & $0.92,1 \cdot 23$ & 0.43 & - & - & - \\
\hline$\geq 350$ & 488 & 62 & 0.90 & $1 \cdot 00$ & & & & & \\
\hline \multicolumn{10}{|c|}{ Malaria parasite density $\left(/ \mathrm{mm}^{3}\right)$} \\
\hline$\geq 1000$ & 111 & 14 & $1 \cdot 07$ & $1 \cdot 19$ & $0 \cdot 98,1 \cdot 43$ & 0.08 & $1 \cdot 22$ & $1 \cdot 04,1 \cdot 43$ & 0.02 \\
\hline $1-999$ & 46 & 6 & 1.06 & $1 \cdot 16$ & $0 \cdot 88,1 \cdot 52$ & $0 \cdot 30$ & $1 \cdot 07$ & $0 \cdot 84,1 \cdot 36$ & 0.59 \\
\hline None & 663 & 80 & 0.90 & $1 \cdot 00$ & & & $1 \cdot 00$ & & \\
\hline \multicolumn{10}{|l|}{$\mathrm{Hb}(\mathrm{g} / \mathrm{dl})$} \\
\hline$<8.5$ & 222 & 27 & 0.93 & $1 \cdot 00$ & $0 \cdot 85,1 \cdot 16$ & 0.95 & - & - & - \\
\hline$\geq 8.5$ & 595 & 73 & 0.93 & $1 \cdot 00$ & & & & & \\
\hline \multicolumn{10}{|c|}{$\begin{array}{l}\text { Mid upper-arm circumference } \\
\quad(\mathrm{cm})\end{array}$} \\
\hline$<22$ & 22 & 6 & $1 \cdot 14$ & $1 \cdot 36$ & $1 \cdot 05,1 \cdot 76$ & 0.02 & $1 \cdot 23$ & $0.96,1.57$ & $0 \cdot 10$ \\
\hline$\geq 22$ & 779 & 94 & 0.90 & $1 \cdot 00$ & & & 1.00 & & \\
\hline \multicolumn{10}{|l|}{ Weight (kg) } \\
\hline$<50$ & 166 & 20 & 0.87 & 0.94 & $0 \cdot 77,1 \cdot 14$ & 0.51 & - & - & - \\
\hline$\geq 50$ & 645 & 80 & 0.94 & $1 \cdot 00$ & & & & & \\
\hline \multicolumn{10}{|l|}{ Ascaris infestation } \\
\hline Yes & 41 & 6 & $1 \cdot 06$ & $1 \cdot 20$ & $0.95,1.52$ & $0 \cdot 12$ & $1 \cdot 15$ & $0.94,1.42$ & $0 \cdot 18$ \\
\hline No & 654 & 94 & 0.88 & $1 \cdot 00$ & & & 1.00 & & \\
\hline \multicolumn{10}{|c|}{ Abnormal vaginal discharge } \\
\hline Yes & 106 & 13 & 1.03 & $1 \cdot 15$ & $0 \cdot 95,1 \cdot 40$ & $0 \cdot 16$ & $1 \cdot 12$ & $0.93,1.35$ & $0 \cdot 25$ \\
\hline No & 712 & 87 & 0.91 & $1 \cdot 00$ & & & $1 \cdot 00$ & & \\
\hline \multicolumn{10}{|l|}{ Age (years) } \\
\hline$>30$ & 141 & 17 & 0.94 & 0.85 & $0 \cdot 66,1 \cdot 10$ & $0 \cdot 22$ & 0.94 & $0 \cdot 73,1 \cdot 21$ & $0 \cdot 61$ \\
\hline $25-29$ & 263 & 32 & $1 \cdot 01$ & 0.92 & $0 \cdot 73,1 \cdot 15$ & 0.44 & 0.97 & $0.78,1 \cdot 21$ & $0 \cdot 79$ \\
\hline $20-24$ & 328 & 40 & $0 \cdot 80$ & 0.73 & $0.58,0.92$ & 0.007 & $0 \cdot 74$ & $0.60,0.92$ & 0.01 \\
\hline$<20$ & 97 & 11 & $1 \cdot 10$ & $1 \cdot 00$ & & & $1 \cdot 00$ & & \\
\hline \multicolumn{10}{|l|}{ Education } \\
\hline No education & 62 & 7 & $1 \cdot 12$ & $1 \cdot 20$ & $0.91,1.58$ & $0 \cdot 19$ & $1 \cdot 16$ & $0 \cdot 90,1 \cdot 49$ & $0 \cdot 26$ \\
\hline Some education & 767 & 93 & 0.92 & $1 \cdot 00$ & & & $1 \cdot 00$ & & \\
\hline Child predictors & & & & & & & & & \\
\hline Gestational age at b & & & & & & & & & \\
\hline$<34$ & 60 & 7 & $1 \cdot 32$ & $1 \cdot 46$ & $1 \cdot 18,1 \cdot 80$ & 0.0004 & $1 \cdot 24$ & $1 \cdot 02,1 \cdot 50$ & 0.03 \\
\hline$\geq 34$ & 769 & 93 & 0.90 & $1 \cdot 00$ & & & 1.00 & & \\
\hline Birth weight (g) & & & & & & & & & \\
\hline$<2500$ & 78 & 10 & $1 \cdot 25$ & $1 \cdot 39$ & $1 \cdot 12,1 \cdot 74$ & 0.003 & $1 \cdot 26$ & $1 \cdot 04,1 \cdot 54$ & 0.02 \\
\hline$\geq 2500$ & 693 & 90 & $0 \cdot 88$ & $1 \cdot 00$ & & & $1 \cdot 00$ & & \\
\hline & $\begin{array}{r}\text { Child } \\
\text { a }\end{array}$ & $\begin{array}{l}\text { onths } \\
\text { sk }\end{array}$ & & & & & & & \\
\hline HIV-infected§ & & & & & & & & & \\
\hline Yes & & & $1 \cdot 37$ & $1 \cdot 70$ & $1 \cdot 47,1 \cdot 97$ & $<0.0001$ & $1 \cdot 61$ & $1 \cdot 40,1 \cdot 85$ & $<0.0001$ \\
\hline No & & & 0.84 & $1 \cdot 00$ & & & 1.00 & & \\
\hline Malaria§ & & & & & & & & & \\
\hline Yes & & & $1 \cdot 34$ & $1 \cdot 52$ & $1 \cdot 25,1 \cdot 84$ & $<0.0001$ & $1 \cdot 52$ & $1 \cdot 25,1 \cdot 85$ & $<0.0001$ \\
\hline No & & & 0.90 & $1 \cdot 00$ & & & $1 \cdot 00$ & & \\
\hline
\end{tabular}

*From separate univariate models using generalized estimating equations with a binomial distribution and a log link.

tFrom separate multivariate models using generalized estimating equations with a binomial distribution and a log link. The multivariate model included all the variables in the column except gestational age at birth and the following additional variables: maternal trial regimen, daily per capita amount of money spent on food and age child started semi-solid foods.

fln an additional model gestational age at birth replaced birth weight.

§HIV infection and malaria were included as time-dependent covariates. 
The risk of developing anaemia in the children was increased if their mothers had a malaria parasite density $\geq 1000 / \mathrm{mm}^{3}$ during pregnancy compared with no parasites (RR 1.22; 95\% CI 1.04, 1.43). HIV infection in the child and malaria in the child were also independently associated with increased risk of anaemia (HIV infection: RR 1.61; 95\% CI $1 \cdot 40,1 \cdot 85$ and malaria: RR 1.52; $95 \%$ CI $1 \cdot 25,1 \cdot 85$ ).
We also examined anaemia suggestive of Fe deficiency in the children based on $\mathrm{Hb}$ and erythrocyte morphology and found that maternal CD4 cell count $<350$ cells $/ \mathrm{mm}^{3}$ during pregnancy was associated with a significantly increased risk (RR 1.58; 95\% CI 1·05, 2.37; Table 2). The risk of anaemia suggestive of Fe deficiency was also increased if the child was HIV-infected or had malaria.

Table 2 Maternal and child factors in relation to anaemia suggestive of iron deficiency among children born to HIV-infected women, Dar es Salaam, Tanzania

\begin{tabular}{|c|c|c|c|c|c|c|c|c|c|}
\hline & \multicolumn{2}{|c|}{ Children at risk } & \multirow{2}{*}{$\begin{array}{l}\text { Anaemia rate } \\
\text { (/child-year) }\end{array}$} & \multirow{2}{*}{$\begin{array}{l}\text { Univariate } \\
\text { relative risk* }\end{array}$} & \multirow[b]{2}{*}{$95 \% \mathrm{Cl}$} & \multirow[b]{2}{*}{$P$ value* } & \multirow{2}{*}{$\begin{array}{l}\text { Adjusted } \\
\text { relative riskt }\end{array}$} & \multirow[b]{2}{*}{$95 \% \mathrm{Cl}$} & \multirow[b]{2}{*}{$P$ valuet } \\
\hline & $n$ & $\%$ & & & & & & & \\
\hline \multicolumn{10}{|l|}{ Maternal predictors } \\
\hline \multicolumn{10}{|l|}{ HIV disease stage } \\
\hline$\geq 2$ & 158 & 19 & $0 \cdot 17$ & $1 \cdot 36$ & $0 \cdot 83,2 \cdot 21$ & $0 \cdot 22$ & - & - & - \\
\hline 1 & 666 & 81 & $0 \cdot 13$ & $1 \cdot 00$ & & & & & \\
\hline \multicolumn{10}{|c|}{ CD4 cell count $\left(/ \mathrm{mm}^{3}\right)$} \\
\hline$<350$ & 293 & 38 & $0 \cdot 18$ & $1 \cdot 59$ & $1 \cdot 05,2 \cdot 41$ & 0.03 & $1 \cdot 58$ & $1 \cdot 05,2 \cdot 37$ & 0.03 \\
\hline$\geq 350$ & 488 & 62 & $0 \cdot 11$ & $1 \cdot 00$ & & & $1 \cdot 00$ & & \\
\hline \multicolumn{10}{|c|}{ Malaria parasite density $\left(/ \mathrm{mm}^{3}\right)$} \\
\hline$\geq 1000$ & 111 & 14 & $0 \cdot 21$ & $1 \cdot 68$ & $1 \cdot 03,2 \cdot 76$ & 0.04 & $1 \cdot 41$ & $0 \cdot 88,2 \cdot 26$ & $0 \cdot 15$ \\
\hline $1-999$ & 46 & 6 & $0 \cdot 15$ & $1 \cdot 27$ & $0.48,3.41$ & 0.63 & 0.92 & $0 \cdot 41,2 \cdot 11$ & 0.85 \\
\hline None & 663 & 80 & 0.12 & $1 \cdot 00$ & & & $1 \cdot 00$ & & \\
\hline \multicolumn{10}{|l|}{$\mathrm{Hb}(\mathrm{g} / \mathrm{dl})$} \\
\hline$<8.5$ & 222 & 27 & $0 \cdot 16$ & $1 \cdot 20$ & $0.78,1 \cdot 83$ & 0.41 & - & - & - \\
\hline$\geq 8.5$ & 595 & 73 & 0.14 & $1 \cdot 00$ & & & & & \\
\hline \multicolumn{10}{|c|}{$\begin{array}{l}\text { Mid upper-arm circumference } \\
\quad(\mathrm{cm})\end{array}$} \\
\hline$<22$ & 22 & 6 & $0 \cdot 26$ & $2 \cdot 09$ & $1 \cdot 09,4 \cdot 00$ & 0.03 & $1 \cdot 51$ & $0.85,2.67$ & $0 \cdot 16$ \\
\hline$\geq 22$ & 779 & 94 & 0.12 & $1 \cdot 00$ & & & $1 \cdot 00$ & & \\
\hline \multicolumn{10}{|l|}{ Weight (kg) } \\
\hline$<50$ & 166 & 20 & 0.14 & $1 \cdot 20$ & $0.75,1.91$ & 0.44 & - & - & - \\
\hline$\geq 50$ & 645 & 80 & 0.13 & $1 \cdot 00$ & & & & & \\
\hline \multicolumn{10}{|l|}{ Ascaris infestation } \\
\hline Yes & 41 & 6 & $0 \cdot 11$ & 0.91 & $0 \cdot 38,2 \cdot 14$ & 0.82 & - & - & - \\
\hline No & 654 & 94 & $0 \cdot 12$ & $1 \cdot 00$ & & & & & \\
\hline \multicolumn{10}{|c|}{ Abnormal vaginal discharge } \\
\hline Yes & 106 & 13 & $0 \cdot 11$ & 0.86 & $0 \cdot 46,1 \cdot 60$ & 0.63 & - & - & - \\
\hline No & 712 & 87 & $0 \cdot 14$ & $1 \cdot 00$ & & & & & \\
\hline \multicolumn{10}{|l|}{ Age (years) } \\
\hline$>30$ & 141 & 17 & $0 \cdot 14$ & 0.82 & $0 \cdot 39,1 \cdot 71$ & 0.59 & 0.93 & $0.51,1.53$ & $0 \cdot 84$ \\
\hline 25-29 & 263 & 32 & $0 \cdot 11$ & 0.62 & $0 \cdot 32,1 \cdot 21$ & $0 \cdot 16$ & $0 \cdot 80$ & $0.45,1 \cdot 42$ & 0.44 \\
\hline $20-24$ & 328 & 40 & $0 \cdot 15$ & 0.86 & $0.46,1 \cdot 61$ & 0.64 & 0.88 & $0 \cdot 47,1 \cdot 84$ & 0.65 \\
\hline$<20$ & 97 & 11 & $0 \cdot 17$ & $1 \cdot 00$ & & & $1 \cdot 00$ & & \\
\hline \multicolumn{10}{|l|}{ Education } \\
\hline No education & 62 & 7 & 0.22 & $1 \cdot 67$ & $0 \cdot 82,3 \cdot 43$ & $0 \cdot 16$ & $1 \cdot 36$ & $0.73,2 \cdot 57$ & 0.34 \\
\hline Some education & 767 & 93 & $0 \cdot 13$ & $1 \cdot 00$ & & & $1 \cdot 00$ & & \\
\hline Child predictors & & & & & & & & & \\
\hline Gestational age at & & & & & & & & & \\
\hline$<34$ & 60 & 7 & 0.36 & 2.93 & $1 \cdot 65,5 \cdot 20$ & 0.0003 & $2 \cdot 03$ & $1 \cdot 11,3 \cdot 69$ & 0.02 \\
\hline$\geq 34$ & 769 & 93 & $0 \cdot 12$ & $1 \cdot 00$ & & & $1 \cdot 00$ & & \\
\hline Birth weight (g) & & & & & & & & & \\
\hline$<2500$ & 78 & 10 & 0.34 & 3.03 & $1 \cdot 82,5 \cdot 07$ & $<0.0001$ & $2 \cdot 62$ & $1 \cdot 60,4 \cdot 26$ & 0.0001 \\
\hline$\geq 2500$ & 693 & 90 & $0 \cdot 11$ & $1 \cdot 00$ & & & $1 \cdot 00$ & & \\
\hline & Child-r & nths at & & & & & & & \\
\hline HIV-infected $\S$ & & & & & & & & & \\
\hline Yes & & & $0 \cdot 27$ & $2 \cdot 53$ & $1 \cdot 69,3 \cdot 79$ & $<0.0001$ & $2 \cdot 37$ & $1 \cdot 59,3 \cdot 55$ & $<0.0001$ \\
\hline No & & & 0.11 & $1 \cdot 00$ & & & $1 \cdot 00$ & & \\
\hline Malaria§ & & & & & & & & & \\
\hline Yes & & 31 & 0.33 & $2 \cdot 74$ & $1 \cdot 73,4 \cdot 33$ & $<0.0001$ & $2 \cdot 85$ & $1 \cdot 74,4 \cdot 67$ & $<0.0001$ \\
\hline No & & & 0.12 & $1 \cdot 00$ & & & $1 \cdot 00$ & & \\
\hline
\end{tabular}

*From separate univariate models using generalized estimating equations with a binomial distribution and a log link.

tFrom separate multivariate models using generalized estimating equations with a binomial distribution and a log link. The multivariate model included all the variables in the column except gestational age at birth and the following additional variables: maternal trial regimen, daily per capita amount of money spent on food and age child started semi-solid foods.

$\ddagger$ In an additional model gestational age at birth replaced birth weight.

$\S$ HIV infection and malaria were included as time-dependent covariates. 
Next we examined anaemia in the children as a timedependent predictor of child mortality to 24 months of age. There were a total of 177 deaths over 15472 childmonths of follow-up. Anaemia suggestive of Fe deficiency was associated with a statistically significant twofold increased risk of death in the total cohort (hazard ratio (HR) 1.99; 95\% CI 1.06, 3.72; Table 3). Child anaemia defined as $\mathrm{Hb}<8.5 \mathrm{~g} / \mathrm{dl}$ did not show a statistically significant association with mortality overall (HR 1.38; $95 \%$ CI $0 \cdot 95,2 \cdot 01)$. However, $\mathrm{Hb}<8.5 \mathrm{~g} / \mathrm{dl}$ was associated with a borderline statistically significant increased risk of death in the HIV-uninfected subgroup (HR 1.86; 95\% CI $0 \cdot 96,3 \cdot 61 ; P=0 \cdot 07$; Table 4 ).

\section{Discussion}

We found a positive association between advanced maternal HIV disease stage during pregnancy defined clinically as per WHO criteria and development of childhood anaemia $(\mathrm{Hb}<8 \cdot 5 \mathrm{~g} / \mathrm{dl})$. Maternal CD4 cell count $<350$ cells $/ \mathrm{mm}^{3}$ during pregnancy was associated with development of anaemia suggestive of Fe deficiency in children in the first two years of life. There were fewer children classified as having Fe-deficiency anaemia and the smaller number of 'events' in the present analysis could be a potential reason why maternal HIV disease stage did not show a statistically significant association even though the point estimate from the univariate analysis showed an increased risk. Advanced clinical and immunological maternal HIV disease during pregnancy has been shown to be associated with increased risk of mortality in children ${ }^{(17-22)}$. Advanced maternal HIV disease could interfere with the transfer of Fe that occurs from the mother to the fetus and also fetal haematopoiesis is affected by maternal HIV infection ${ }^{(23-25)}$. Placental malaria has been shown to be associated with child anaemia at 2 months ${ }^{(26)}$ and 6 months of age ${ }^{(27)}$ among

Table 3 Association of child anaemia with overall mortality among children born to HIV-infected women, Dar es Salaam, Tanzania

\begin{tabular}{|c|c|c|c|c|c|c|c|c|}
\hline Child anaemia & $\begin{array}{l}\text { Child-months } \\
\text { at risk }\end{array}$ & $\begin{array}{l}\text { No. of } \\
\text { deaths }\end{array}$ & $\begin{array}{c}\text { Hazard } \\
\text { ratio** } \\
\text { (univariate) }\end{array}$ & $95 \% \mathrm{Cl}$ & $P$ value* & $\begin{array}{l}\text { Hazard ratiot } \\
\text { (adjusted) }\end{array}$ & $95 \% \mathrm{Cl}$ & $P$ valuet \\
\hline \multicolumn{9}{|l|}{$\mathrm{Hb}(\mathrm{g} / \mathrm{dl})$} \\
\hline $\begin{array}{l}<8.5 \\
\geq 8.5\end{array}$ & $\begin{array}{r}3590 \\
11882\end{array}$ & $\begin{array}{r}43 \\
134\end{array}$ & $\begin{array}{l}1 \cdot 30 \\
1 \cdot 00\end{array}$ & $0 \cdot 91,1 \cdot 86$ & $0 \cdot 16$ & $\begin{array}{l}1 \cdot 38 \\
1 \cdot 00\end{array}$ & $0 \cdot 95,2 \cdot 01$ & $0 \cdot 10$ \\
\hline \multicolumn{9}{|c|}{$\begin{array}{l}\mathrm{Hb}<8.5 \mathrm{~g} / \mathrm{dl} \text { and hypochromic } \\
\text { microcytosis }\end{array}$} \\
\hline Yes & 548 & 12 & $2 \cdot 34$ & $1 \cdot 29,4 \cdot 23$ & 0.005 & 1.99 & $1 \cdot 06,3 \cdot 72$ & 0.03 \\
\hline No & 14924 & 165 & $1 \cdot 00$ & & & $1 \cdot 00$ & & \\
\hline
\end{tabular}

*From separate univariate Cox proportional hazards models with anaemia as a time-dependent covariate.

tFrom separate multivariate Cox proportional hazards models with anaemia as a time-dependent covariate. The multivariate models also included the following adjusting variables, with indicator variables for individuals missing data on a particular variable: maternal trial regimen, CD4 cell count during pregnancy, education, daily per capita amount of money spent on food and the child variables of birth weight, age started semi-solid foods, time-varying CD4 cell counts, malaria parasitaemia and HIV infection status.

Table 4 Association of child anaemia with mortality stratified by HIV status among children born to HIV-infected women, Dar es Salaam, Tanzania

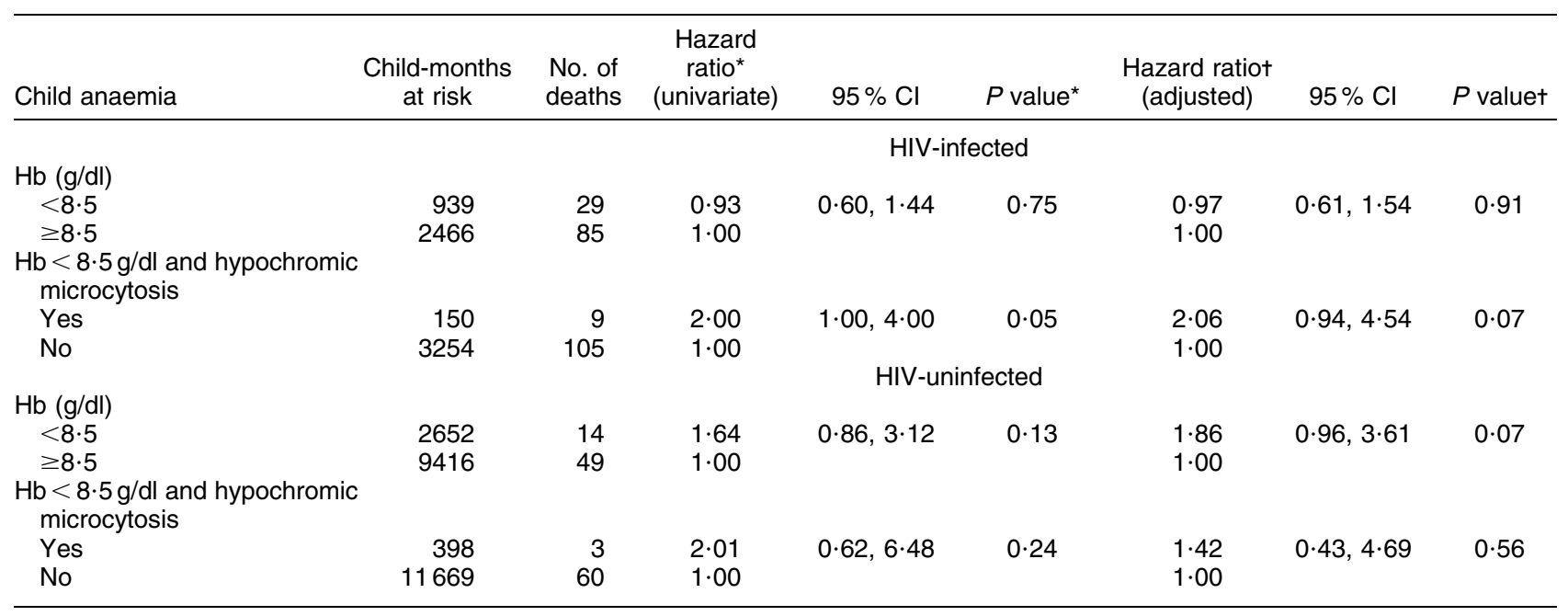

*From separate univariate Cox proportional hazards models with anaemia as a time-dependent covariate.

†From separate multivariate Cox proportional hazards models with anaemia as a time-dependent covariate. The multivariate models also included the following adjusting variables, with indicator variables for individuals missing data on a particular variable: maternal trial regimen, CD4 cell count during pregnancy, education, daily per capita amount of money spent on food and the child variables of birth weight, age started semi-solid foods, time-varying CD4 cell counts and malaria parasitaemia. 
children born to HIV-uninfected mothers. Our study extends these findings by demonstrating that the increased risk of child anaemia due to maternal malaria during pregnancy may extend into the second year of life among children born to HIV-infected women.

These results are in agreement with earlier smaller studies from sub-Saharan Africa, which reported a high prevalence of anaemia among children with HIV infection who were not on antiretroviral therapy ${ }^{(28-31)}$ and noted $\mathrm{HIV}$ infection to be associated with a decrease in $\mathrm{Hb}$ levels over time ${ }^{(32)}$. HIV infection in children could cause anaemia by interfering with haematopoiesis or absorption of Fe and the presence of opportunistic infections ${ }^{(33)}$.

We noted a positive association between anaemia in the children and mortality over the period of follow-up. However, only anaemia suggestive of Fe deficiency was statistically significantly related to mortality. In Uganda, antiretroviral-naïve HIV-infected children with anaemia $(\mathrm{Hb}<9 \mathrm{~g} / \mathrm{dl})$ at 9 months had a $59 \%$ increased risk of death by 36 months of age ${ }^{(34)}$ and anaemia was also a predictor of disease progression among HIV-infected children in another study ${ }^{(35)}$. A recently published metaanalysis has shown an association between $\mathrm{Hb}$ levels and mortality among antiretroviral-naïve HIV-infected children $^{(36)}$. The children in our study were of a younger age group than those in the two studies mentioned. The study from Uganda reported the association between one measure of $\mathrm{Hb}$ and mortality whereas our study captured $\mathrm{Hb}$ levels over a longer period of time by using multiple measurements available for each child from birth to 2 years of age. Two separate Fe and folic acid supplementation trials were carried out among children aged 1-36 months born to women who were presumably HIVnegative ${ }^{(37,38)}$. In the trial from Pemba ${ }^{(37)}$, adverse events due to malaria-related causes and infection-related causes were increased in the children who received Fe and folic acid supplementation compared with those who received placebo. In the trial from Nepal there was no effect of supplementation on mortality ${ }^{(38)}$. The children enrolled in these studies are different from those studied in the present study, which only included children born to HIVinfected women. Moreover, our results are in agreement with those reported among the subgroup of children who had Fe deficiency and anaemia in the Pemba trial, which demonstrated a protective effect of $\mathrm{Fe}$ and folic acid supplementation against serious adverse events in that subgroup of children ${ }^{(37)}$. Also, in the Nepal trial, there were statistically non-significant reductions in respiratory and diarrhoea morbidity in the group that received Fe and folic acid as compared with placebo ${ }^{(38)}$.

There are some potential limitations in our study methodology. Gestational age was determined using maternal recall of last menstrual period, which might not be totally accurate. Due to logistical reasons $\mathrm{Hb}$ measurements were done using two different methods using CBC5 Coulter counter and colorimeter. However, both these methods give comparable results ${ }^{(39)}$. Biochemical measurements such as serum ferritin would have given a more accurate diagnosis of Fe-deficiency anaemia; however, erythrocyte morphology is also used to make a clinical diagnosis of Fe-deficiency anaemia in the absence of more definitive biochemical measurements ${ }^{(40)}$. We did not have detailed information on the diet of the children and the role it may have played in their development of anaemia. However, we controlled for quality of diet using as a proxy indicator, the daily per capita amount of money spent on food.

The results from the present study have important public health implications for countries with a high prevalence of HIV infection and a high burden of anaemia among children. Childhood anaemia is known to be associated with impaired psychomotor and cognitive development ${ }^{(7,8)}$. The results from our study show that anaemia among children born to HIV-infected women, especially when related to Fe deficiency, is also associated with increased risk of mortality. HIV-exposed children receiving follow-up care within programmes for prevention of mother-to-child transmission of HIV and paediatric AIDS should receive a comprehensive package of child survival interventions including screening and treatment for anaemia. Assessment of HIV-positive pregnant women for antiretroviral therapy for their own health, including clinical staging of HIV disease and CD4 cell counts, should be scaled up on a priority basis; only about $12 \%$ of HIV-positive pregnant women received this assessment in $2007^{(41)}$. These interventions, when implemented at scale, can have a significant impact on child survival and development in sub-Saharan Africa.

\section{Acknowledgements}

This work was supported by the National Institute of Child Health and Human Development (NICHD R01 32257), the Fogarty International Center (NIH D43 TW00004) and the Harvard School of Public Health. We declare that we have no conflict of interest. A.C. analysed and interpreted the data and wrote the initial draft of the manuscript. R.J.B. provided statistical guidance in data analyses. R.K. assisted in interpretation of the results. D.J.H. assisted in the design of the Tanzania Vitamin Supplementation Trial and in data interpretation. G.I.M. and W.W.F. are principal investigators of the Tanzania Vitamin Supplementation Trial. All co-authors participated in manuscript preparation. We gratefully acknowledge the contributions of the field staff in data collection and the women and children enrolled in the study.

\section{References}

1. Ezzati M, Lopez AD, Rodgers A, Vander Hoorn S \& Murray CJ; Comparative Risk Assessment Collaborating Group 
(2002) Selected major risk factors and global and regional burden of disease. Lancet 360, 1347-1360.

2. United Nations Administrative Committee on Coordination, Sub-committee on Nutrition (2000) Fourth Report on the World Nutrition Situation: Nutrition Throughout the Life-cycle. Geneva: UN Press.

3. Belperio PS \& Rhew DC (2004) Prevalence and outcomes of anemia in individuals with human immunodeficiency virus: a systematic review of the literature. Am J Med $\mathbf{1 1 6}$ Suppl. 7A, 27S-43S.

4. DeMaeyer E \& Adiels-Tegman M (1985) The prevalence of anemia in the world. World Health Stat $Q \mathbf{3 8}, 302-316$.

5. Stoltzfus R, Mullany L \& Black RE (2004) Iron deficiency anemia. In Comparative Quantification of Health Risks: The Global and Regional Burden of Disease due to 25 Selected Major Risk Factors, pp. 163-209 [M Ezzati, AD Lopez, A Rodgers and CJL Murray, editors]. Geneva: WHO.

6. Stoltzfus RJ \& Dreyfus MJ (1998) Guidelines for the Use of Iron Supplements to Prevent and Treat Iron Deficiency Anemia. Washington, DC: ILSI Press.

7. Lozoff B, Brittenham GM, Wolf AW, McClish DK, Kuhnert PM, Jimenez E, Jimenez R, Mora LA, Gomez I \& Krauskoph D (1987) Iron deficiency anemia and iron therapy effects on infant development test performance. Pediatrics $\mathbf{7 9}$, 981-985.

8. Beard J (2001) Iron biology in immune function, muscle metabolism and neuronal functioning. J Nutr 131, 2 Suppl., 568S-580S.

9. Chandra RK (1973) Reduced bactericidal activity of polymorphs in iron deficiency. Arch Dis Child 48, 864-867.

10. Bhaskaram P \& Reddy V (1975) Cell mediated immunity in iron- and vitamin-deficient children. $\mathrm{Br}$ Med $J$ iiii, 522-524.

11. Mocroft A, Kirk O, Barton S, Dietrich M, Proenca R, Colebunders R, Pradier C, dArminio Monforte A, Ledergerber B \& Lundgren JD (1999) Anaemia is an independent predictive marker for clinical prognosis in HIV-infected patients from across Europe. EuroSIDA Study Group. AIDS 13, 943-950.

12. O'Brien ME, Kupka R, Msamanga GI, Saathoff E, Hunter DJ \& Fawzi WW (2005) Anemia is an independent predictor of mortality and immunologic progression of disease among women with HIV in Tanzania. J Acquir Immun Defic Syndr 40, 219-225.

13. Fawzi WW, Msamanga GI, Spiegelman D et al. (1998) Randomised trial of effects of vitamin supplements on pregnancy outcomes and T cell counts in HIV-1-infected women in Tanzania. Lancet 351, 1477-1482.

14. Lohman TG, Roche AF \& Martorell R (editors) (1988) Anthropometric Standardization Reference Manual. Champaign, IL: Human Kinetics Publishing.

15. Diggle P, Liang K \& Zeger S (1994) Analysis of Longitudinal Data. London: Oxford University Press.

16. Miettinen OS (1985) Theoretical Epidemiology. New York: John Wiley and Sons.

17. Blanche S, Rouzioux C, Moscato ML et al. (1989) A prospective study of infants born to women seropositive for human immunodeficiency virus type 1 . HIV Infection in Newborns French Collaborative Study Group. N Engl J Med 320, 1643-1648.

18. Blanche S, Mayaux MJ, Rouzioux C et al. (1994) Relation of the course of HIV infection in children to the severity of the disease in their mothers at delivery. $N$ Engl J Med 330, 308-312.

19. Lambert G, Thea DM, Pliner V et al. (1997) Effect of maternal CD4+ cell count, acquired immunodeficiency syndrome, and viral load on disease progression in infants with perinatally acquired human immunodeficiency virus type 1 infection. New York City Perinatal HIV Transmission Collaborative Study Group. J Pediatr 130, 890-897.
20. Abrams EJ, Wiener J, Carter R, Kuhn L, Palumbo P, Nesheim S, Lee F, Vink P \& Bulterys M; Perinatal AIDS Collaborative Transmission Study (PACTS) Group (2003) Maternal health factors and early pediatric antiretroviral therapy influence the rate of perinatal HIV-1 disease progression in children. AIDS 17, 867-877.

21. Chearskul S, Chotpitayasunondh T, Simonds RJ et al.; Bangkok Collaborative Perinatal HIV Transmission Study Group (2002) Survival, disease manifestation and early predictors of disease progression among children with perinatal human immunodeficiency virus infection in Thailand. Pediatrics 110, 25-30.

22. Chatterjee A, Bosch RJ, Hunter DJ, Fataki MR, Msamanga GI \& Fawzi WW (2007) Maternal disease stage and child undernutrition in relation to mortality among children born to HIV-infected women in Tanzania. J Acquir Immun Defic Syndr 46, 599-606.

23. Widdowson EM \& Spray CM (1951) Chemical development in utero. Arch Dis Child 26, 205-214.

24. Singla PN, Gupta VK \& Agarwal KN (1985) Storage iron in human fetal organs. Acta Pediatr Scand 74, 701-706.

25. Burnstein Y, Rashbaum WK, Hatch WC, Calvelli T, Golodner M, Soeiro R \& Lyman WD (1992) Alterations in human fetal erythropoiesis are associated with maternal HIV infection. Pediatr Res 32, 155-159.

26. Reed SC, Wirima JJ \& Stekeete RW (1994) Risk factors for anemia in young children in rural Malawi. Am J Trop Med Hyg 51, 170-174.

27. Cornet M, Le Hesran JY, Fievet N, Cot M, Personne P, Gounoue R, Beyeme M \& Deloron P (1998) Prevalence of and risk factors for anemia in young children in southern Cameroon. Am J Trop Med Hyg 58, 606-611.

28. Adewuyi J \& Chitsike I (1994) Hematologic features of the human immunodeficiency virus (HIV) infection in black children in Harare. Cent Afr J Med 40, 333-336.

29. Shaffer N, Hedberg K, Davachi F, Lyamba B, Breman JG, Masisa OS, Behets F, Hightower A \& Nguyen-Dinh P (1990) Trends and risk factors for HIV-1 seropositivity among outpatient children, Kinshasa, Zaire. AIDS 4, 1231-1236.

30. Totin D, Ndugwa C, Mmiro F, Perry RT, Jackson JB \& Semba RD (2002) Iron deficiency anemia is highly prevalent among human immunodeficiency virus-infected and uninfected infants in Uganda. J Nutr 132, 423-429.

31. Eley BS, Sive AA, Shuttleworth M \& Hussey GD (2002) A prospective, cross-sectional study of anemia and peripheral iron status in antiretroviral naïve, HIV-infected children in Cape Town, South Africa. BMC Infect Dis $\mathbf{2}, 3$.

32. Villamor E, Mbise R, Spiegelman D, Ndossi G \& Fazwi Ww (2000) Vitamin A supplementation and other predictors of anemia among children from Dar es Salaam, Tanzania. Am J Trop Med Hyg 62, 590-597.

33. Semba RD \& Gray GE (2001) The pathogenesis of anemia during human immunodeficiency virus infection. J Invest Med 49, 225-239.

34. Clark TD, Mmiro F, Ndugwa C, Perry RT, Jackson JB, Melikian G \& Semba RD (2002) Risk factors and cumulative incidence of anaemia among human immunodeficiency virusinfected children in Uganda. Ann Trop Pediatr 22, 11-17.

35. Ellaurie M, Burns ER \& Rubinstein M (1990) Hematologic manifestations in pediatric HIV infection: severe anemia as a prognostic factor. Am J Pediatr Hematol Oncol 12, 449-453.

36. Cross Continents Collaborative for Kids (3Cs4kids) Analysis and Writing Committee (2008) Markers for predicting mortality in untreated HIV-infected children in resource-limited settings: a meta-analysis. AIDS 22, 97-105.

37. Sazawal S, Black RE, Ramsan M et al. (2006) Effects of routine prophylactic supplementation with iron and folic 
acid on admission to hospital and mortality in preschool children in a high malaria transmission setting: communitybased, randomized, placebo-controlled trial. Lancet 367, 133-143.

38. Tielsch JM, Khatry SK, Stotlzfus RJ, Katz J, LeClerq SC, Adhikari R, Mullany LC, Shresta S \& Black RE (2006) Effect of routine prophylactic supplementation with iron and folic acid on preschool child mortality in southern Nepal: community-based cluster-randomized, placebo-controlled trial. Lancet 367, 144-152.
39. Pinkerton PH, Spence I, Ogilvie JC, Ronald WA, Marchant P \& Ray PK (1970) An assessment of the Coulter counter model S. J Clin Pathol 23, 68-76.

40. Massawe SN, Urassa EN, Mmari M, Ronquist G, Lindmark G \& Nystrom L (1999) The complexity of pregnancy anemia in Dar-es-Salaam. Gynecol Obstet Invest 47, 76-82.

41. World Health Organization/Joint United Nations Programme on HIV/AIDS/UNICEF (2008) Towards Universal Access: Scaling Up Priority HIV/AIDS Interventions in the Health Sector: Progress Report 2008. Geneva: WHO. 